\title{
Información desagregada, elemento clave para la toma de decisiones
}

\section{Disaggregated information, key to decisions}

\author{
Garrocho Rangel, Carlos (20 i i), Población flotante, población \\ EN MOVIMIENTO: CONCEPTOS CLAVE Y MÉTODOS DE ANÁLISIS EXITOSOS, \\ Consejo Nacional de Población-El Colegio Mexiquense, a.c., \\ MÉXICO, 266 PP., ISBN: 978-607-427-I03-4.
}

La información oportuna representa el insumo fundamental para atender diversas problemáticas y formular estrategias adecuadas para resolverlas. Por lo que el quehacer de cualquier persona encargada de tomar decisiones depende de contar con información clara y oportuna para trabajar con certidumbre y poder definir estrategias que logren cumplir sus metas. Pero ¿qué sucede cuando la información que se tiene es genérica y no permite conocer a fondo la problemática?

Un estratega serio tendrá que ordenar los estudios necesarios para tomar una postura ante el fenómeno a enfrentar, lo que implica disponer de tiempo para que se realicen los estudios necesarios y retrasar las decisiones.

En esta situación se han encontrado directivos de diversas áreas, una de ellas es la de vialidad y transporte, pues al pretender mejorar sus condiciones y no contar con información desagregada referente a movilidad cotidiana, por ejemplo, se han mandado realizar estudios origen-destino que proporcionen la información que requieren para valorar las estrategias a seguir; por consiguiente, queda en espera el planteamiento de soluciones. Situaciones de este tipo han tenido que enfrentar diferentes organismos gubernamentales, como en los casos del estudio realizado por el Instituto Nacional de Estadística, Geografía e Informática (INEGI) a petición del Gobierno del Distrito Federal (GDF) y del Estado de México en 2007, con la intención de obtener información referente a la movilidad de los residentes de la Zona Metropolitana del Valle de México; así como el Estudio de movilidad urbana de Nuevo Laredo, Tamaulipas, en 2009, elaborado por el Instituto Municipal de Investigación, Planeación y Desarrollo Urbano (Impladu, 2009), con el objetivo de conocer los patrones de viajes y forma en que la población utiliza la infraestructura vial y medios de transporte dentro de esa ciudad.

Para entender mejor los sucesos que tienen que afrontar, revisemos el caso del estudio del INEGi antes citado. Como antecedente de este proyecto tenemos que en 1983, la Coordinación General de Transporte del 
Departamento del Distrito Federal (a partir de estimaciones de El Colegio de México) realizó un Estudio origen-destino (EOD-83), para el cual se consideró a las 16 delegaciones del Distrito Federal (DF) y a 89 localidades mayores de 1,000 habitantes y/o las cabeceras de 27 municipios del Estado de México.

Once años más tarde, el INEGI levantó la Encuesta de origen y destino de los viajes de los residentes del Área Metropolitana de la Ciudad de México (EOD-94). En aquella ocasión se captó información de las 16 delegaciones del DF y 28 municipios conurbados del Estado de México (Connolli, 2009).

El 15 de noviembre de 2005, la Cámara de Diputados del H. Congreso de la Unión aprobó el Presupuesto de egresos de la federación para el ejercicio fiscal 2006, en el cual se destinaron recursos para el Fondo Metropolitano de Proyectos de Impacto Ambiental en el Valle de México en el apartado "Obras de infraestructura vial y de comunicaciones", para que bajo la responsabilidad del GDF se elaborara la Encuesta origen-destino de la Zona Metropolitana del Valle de México (zмvм) 2007, con la cual se logró:

- Estimar la cantidad de viajes de la población de 6 años y más que se generaron en la ZMvM en días típicos laborables (lunes a viernes, excluyendo periodos vacacionales), así como los motivos por los cuales se realizaron.

- Conocer los modos de transporte empleados y los transbordos que se requerían para llegar al destino final (viajes y tramos de viajes).

- Captar el tiempo y el costo de transportación en un día de viaje.

- Detectar las horas de mayor afluencia de viajes.

- Relacionar las características sociodemográficas de los miembros del hogar con los viajes que realizaban.

- Proveer información que permitiera calibrar los modelos de demanda y oferta de los sistemas de transporte de la ZMVM (INEGI, 2007).

De esta manera se pudo contar con datos desagregados para determinar la movilidad de los residentes de la ZMvM, beneficiando así el proceso de planeación de transporte y vialidades que llevan a cabo las dependencias correspondientes de los gobiernos del Distrito Federal y del Estado de México.

Sin embargo, la obtención de la información fue resultado de un proceso de varios años de gestión, ya que desde 2005 se iniciaron los trabajos para obtener recursos suficientes para realizar el estudio que fi- 
nalmente se entregó en 2007. Este periodo representó una perdida de tiempo valioso en la generación de soluciones a problemas apremiantes.

De acuerdo con Carlos Garrocho, esta falta de información no es exclusiva del caso mexicano, otros países se encuentran en una situación similar, donde la toma de decisiones relacionadas con la planeación debe posponerse hasta contar con estudios que les den los datos necesarios para plantear estrategias, como es el caso de la Encuesta origen destino de viajes de Santiago de Chile, que la Universidad Católica (2002), por encargo del Ministerio de Planeación y Cooperación (Mideplan), llevó a cabo para conocer los viajes y características de los pasajeros que se transportaban dentro de la ciudad.

Los problemas que enfrentan las ciudades son urgentes, por lo que es preocupante tener que esperar a que se genere información, cuando es apremiante ofrecer estrategias de solución a los problemas que enfrentan nuestras ciudades.

En este contexto, Carlos Garrocho propone una metodología clara para forjar información clave para ir de la planeación correctiva a una propositiva. Así, presenta una propuesta interesante para estimar la población flotante, convencido de la importancia que tiene para los agentes gubernamentales y económicos el conocimiento de la distribución demográfica sobre el territorio para la adecuada toma de decisiones.

Garrocho inicia su exposición aclarando la importancia de realizar una estimación adecuada de la población flotante, para lo cual explica desde el concepto genérico de la movilidad de la población, cómo de ésta se desprende el término de población flotante, hasta los conceptos de migración permanente, temporal y movilidad cotidiana para concluir con la propuesta de una definición de población flotante que permita su análisis.

En el curso de la dilucidación de la población flotante resulta interesante la denuncia que realiza de la situación en que se encuentra la información censal de México, España y China. Muestra cómo la metodología es un punto trascendental para dejar o no al margen la información de la población flotante, ya sea, por ejemplo, por la estructura de las preguntas o por el lugar donde se levanta el censo.

El autor dirige la atención en la definición de las principales modalidades de población flotante, primero bajo los enfoques micro y macro, y después la analiza con respecto a tres dimensiones: espacial, temporal y tipo de actividad, para concluir que los mercados laborales locales se explican, en términos generales, por la movilidad de la población y de manera específica se trata de la complementación de la migración temporal y la movilidad cotidiana. 
La decisión de migrar, vista desde un enfoque micro, se basa en el supuesto clásico de que depende del balance entre costos y beneficios; de hacerlo a nivel macro se da por la existencia de heterogeneidades espaciales, pero el autor va más allá de mostrarnos los enfoques clásicos que expliquen el comportamiento de la toma de decisiones para la migración, expone que los individuos no reaccionan igual ante las diferentes características de cada lugar y que la pobreza no es el factor primordial, ya que la población en extrema pobreza migra menos porque ni siquiera puede realizar la inversión inicial para detonar el proceso migratorio. De igual manera enuncia una serie de patrones reveladores de migración interna en diversas regiones del mundo en desarrollo, como la feminización de la migración.

Concluye la primera parte de su investigación con la identificación de los principales problemas del estudio de la población flotante, la cual se centra en la falta de estadísticas de calidad, o con la ausencia de definiciones conceptuales y operativas, pero que finalmente esto representa un reto para gestar un esquema metodológico viable, por lo que la segunda parte de su libro la dedica a presentar ejemplos metodológicos exitosos para medir a la población flotante y las cargas de población que puedan adaptarse al caso mexicano; para lo cual analiza tres temas: los mercados laborales locales (MLL), la movilidad cotidiana de la población, y la migración temporal.

En el tema de mLl explica a detalle los métodos utilizados para su definición y hace hincapié en las principales dificultades que presenta México para conceptualizarlo, a diferencia de países líderes como Canadá, Estados Unidos y el Reino Unido por la disponibilidad de amplias bases de datos y la experiencia operativa en la materia.

Para el tema de movilidad cotidiana se eligieron los casos exitosos de Canadá, Estados Unidos, el Reino Unido y Holanda, porque cuentan con una metodología reconocida a nivel mundial y porque tienen un alto grado de detalle en estudios de movilidad cotidiana. En estos cuatro casos de estudio se observó que se le concede una gran relevancia al estudio de la población flotante y la delimitación de los MLL para la planeación de regiones y ciudades, situación disímil en el caso mexicano.

El caso canadiense reporta encuestas de movilidad a escala regional y metropolitana, como ejemplo expone la pesquisa Transportation Tomorrow Survey, que cubre la zona metropolitana de Toronto y que se ha realizado de manera quinquenal, la cual contiene información detallada de todos los integrantes de los hogares encuestados, como las características de los cambios de la demanda por viajes que permite analizar la relocalización del empleo, entre otras actividades. 
Para Estados Unidos Garrocho expone la Encuesta National Household Travel Survey, fuente principal de información referente al comportamiento de la movilidad cotidiana en este país. En ella se incluye información sobre tipos, longitud y propósitos de viajes, modos de transporte, frecuencia y generación de viajes por hogar, entre muchas otras variables; información que se actualiza cada siete años en promedio, lo que ha permitido generar diagnósticos y pronósticos más confiables y diseñar modelos de movilidad y transporte más precisos.

Para el Reino Unido expone la encuesta National Travel Survey, que monitorea las tendencias y el comportamiento de los viajes personales dentro de Inglaterra, Escocia, Gales e Irlanda del Norte, de manera permanente, de este modo se conocen los cambios en el patrón de viajes cotidianos y se puede analizar la dinámica de los tiempos de transporte, diseñar modelos de transporte a diferentes escalas geográficas, entre muchos otros usos relacionados con la planeación urbana y regional.

En el caso de Holanda presenta la encuesta Dutch National Travel Survey, que describe el comportamiento de la movilidad de la población holandesa, identifica los patrones de movilidad de grupos clave, según su punto de partida y destino, periodo de movilidad, modo de transporte y propósito del desplazamiento. Esta encuesta es anual y cuenta con información con un alto grado de precisión, ya que contiene registros del lugar de origen y destino para cada viaje, la distancia recorrida, la hora de inicio y fin del viaje, modos de transporte utilizado, propósito del viaje, edad y género de las personas que realizan los viajes.

Después de mostrar las características metodológicas que utilizan estos cuatro casos exitosos, el autor expone el caso mexicano y deja de manifiesto la carencia de estudios de movilidad cotidiana, ya que a pesar de que existen encuestas origen-destino, éstas se han realizado para casos y momentos específicos, por lo que no se cuenta con un trabajo institucional que realice esta labor de manera permanente. Reconoce los estudios del InEgi (2007) y de Nava (2009) para la Zona Metropolitana de la Ciudad de México, pero el primero se dio bajo contrato a solicitud de gobiernos locales para plantear un proyecto de transporte, y el segundo se trata de una tesis de doctorado que tiene una metodología interesante, pero que corre el riesgo de no tener seguimiento en su actualización.

Aunque en este punto habría que recordar que a lo largo del tiempo diversos organismos han realizado estos ejercicios, el caso citado de la ZMVM presenta una secuencia de estudios (Connolli, 2009), que si bien no han conservado una misma metodología, sí manifiestan que hubo una necesidad de información estadística puntual referente al tema de movilidad.

La gran diferencia que encontramos entre los casos extranjeros con los nacionales no radica primordialmente en la metodología, ya que en Méxi- 
co se cuenta con buenos ejemplos, va más en el sentido de valorar la importancia de contar con estudios de movilidad cotidiana para realizar una planeación a largo plazo con mayores posibilidades de éxito, donde el costo de los estudios se contemplen como inversión para el mejor funcionamiento de las ciudades, donde se reduzca la incertidumbre en la toma de decisiones y se trabaje con una planeación prospectiva y no correctiva.

El tercer tema que aborda en la segunda parte de su trabajo es el de migración temporal interna, donde analiza los métodos de recopilación de información de China, Estados Unidos y Nueva Zelanda para ilustrar la complejidad y dimensiones que implica cuantificar este tipo de migración.

El autor analiza que en China se han desarrollado encuestas específicas para este quehacer, pero sin buenos resultados por la falta de homogeneidad de los conceptos que generan ambigüedades en su estimación, la ausencia de conteos diseñados para registrar a la población flotante en las ciudades, las metodologías utilizadas que no han logrado la selección adecuada de los lugares donde se realiza el conteo y la falta de esfuerzos institucionales articulados.

Garrocho argumenta que en este tema Estados Unidos también presentaba una carencia de información, por lo que se suplía con estimaciones anuales, pero que a partir de 2010, a través de The American Community Survey genera información anualizada de características sociales, económicas y demográficas por zona (census tracks), por lo que se perfila que será, a decir de Garrocho, la encuesta más avanzada en el mundo en métodos, cobertura y periodicidad referentes a las características de población.

Finalmente, con respecto a Nueva Zelanda se expone que al ser un país interesado en conocer la distribución espacial de su población, cuenta con una encuesta especializada en el tema llamada Survey of Dynamics and Motivation for Migration in New Zealand, la cual examina la migración y las motivaciones de la población para desplazarse, con lo que se puede analizar la competitividad y capacidades de atracción y retención de población de los diferentes asentamientos de su territorio.

De manera ideal el estudio de la migración temporal debe complementarse con estudios cualitativos en profundidad, como éstos son costosos y poco generalizables es necesario contar con una buena estrategia para recopilar información, por lo que el autor expone varias recomendaciones para estimar la magnitud de la migración temporal, del mismo modo presenta 10 modelos de migración interna para proyectar poblaciones subnacionales como alternativa a los trabajos de investigación que no cuentan con elevados recursos financieros; además muestra una opción para estimar la población de facto, ya que con frecuencia la única información demográfica disponible es con la población de jure. 
Después del profundo análisis que hace el autor de la situación en la que se encuentra no sólo México, sino muchos otros países, en la comprensión del comportamiento de la movilidad de la población y su efecto en el territorio, plantea entre varias propuestas la selección de metodologías internacionales exitosas que puedan aplicarse al caso mexicano, como el de la Encuesta de las dinámicas y motivaciones de la migración en Nueva Zelanda; propone el diseño general de metodologías propias; diseñar esquemas de financiamiento conjunto para llevar a cabo los estudios; evaluar los resultados de las estimaciones; iniciar una etapa de mejora continua, y concluye con la exposición del perfil de una propuesta metodológica con un caso de estudio hipotético, donde comienza definiendo a la población flotante como "la población que utiliza un territorio pero cuyo lugar de residencia habitual es otro. La población flotante, sumada a la población residente, permitirá determinar la carga de población que realmente soporta cada territorio" (p. 231) y lo sintetiza en la siguiente fórmula: $\mathrm{CP}=\mathrm{Pj}+\mathrm{Pij}-\mathrm{Pji}$.

Donde CP es la carga de población que soporta la ciudad, Pj es la población residente en la ciudad, Pij es la población que llega de otra ciudad y Pji es la población que sale de la ciudad en cuestión.

De esta manera, con una definición clara es posible conocer la distribución de la demanda en el territorio, lo que lleva a mejorar la toma de decisiones y aplicación de la planeación estratégica y táctica tanto en el sector público como en el privado.

En conclusión, el libro Población flotante, población en movimiento: conceptos clave y métodos de análisis exitosos es una constante invitación a profundizar y realizar investigaciones detalladas, es un texto que genera inquietudes, nos introduce en el tema de la movilidad de la población, nos hace reconocer su importancia y su situación en el ámbito mundial, nos sensibiliza ante la problemática de la falta de información y utilización de metodologías erróneas y nos motiva al acuerdo de definir conceptos, de crear conciencia, ya que el problema no es sólo del tema de movilidad, sino de la falta de información oportuna.

Es imperante entender la generación de información clave como una inversión para el desarrollo económico y apostar por mantener una base de datos actualizada que permita la toma de medidas oportunas. No se pueden solucionar los problemas territoriales sin planeación, pero no se puede hacer planeación sin información, y ésta no se logra si no se le considera un tema prioritario que sirve de cimiento a la toma de decisiones. 


\section{Bibliografía}

INEGI (Instituto Nacional de Estadística, Geografía e Informática) (2007), Encuesta origen-destino de los residentes de la Zona Metropolitana del Valle de México 2007, INEGi, México.

Connolly, Priscilla (2009), "La pérdida de movilidad”, Ciudades, 81, Red Nacional de Investigación Urbana, Puebla, <http://132.248.9.1: 8991/hevila/e-BIBLAT/CLASE/cla299865.pdf>, 7 de abril de 2012.

Impladu (Instituto Municipal de Investigación, Planeación y Desarrollo Urbano) (2009), "Estudio-metodología de origen y destino. Movilidad urbana", Ayuntamiento Nuevo Laredo 2008-2010-Impladu, México, <http://impladu.gob.mx>, 6 de marzo de 2012.

Nava Emelina (2009), Estructura urbana policéntrica y movilidad exploraciones en torno a la distancia y el tiempo de desplazamiento en el área metropolitana de la Ciudad de México, tesis de doctorado en Urbanismo, Programa de Maestría y Doctorado en Urbanismo, unam, México.

Universidad Católica (2002), "Encuestas origen destino de viajes 20012002, Santiago de Chile”, Universidad Católica-Mideplan, Chile, <http://www.subtrans.gob.cl/subtrans/doc/estadisticasEOD2001_Informe_Difusion.pdf $>, 6$ de marzo de 2012.

Adriana Soledad Espinosa-Flores Universidad Autónoma del Estado de México Correo-e: fad_apou@yahoo.com.mx

Adriana Soledad Espinosa-Flores. Es maestra en ciencias sociales, con especialidad en desarrollo municipal, por El Colegio Mexiquense. Actualmente estudia el doctorado en urbanismo en la Universidad Nacional Autónoma de México; es profesora de la Facultad de Arquitectura y Diseño de la Universidad Autónoma del Estado de México. Su línea de investigación actual es políticas públicas en el desarrollo urbano. Entre sus últimas publicaciones destacan: "Políticas de suelo para vivienda en México", en Colegio de Ingenieros de Caminos, Canales y Puertos, Un nuevo modelo para una nueva época, CICCP, Madrid, CD, (2011); "Adquisición de suelo urbanizable para pobres. Un cambio de política apremiante", en Alfonso Iracheta, Miguel Ángel García y Rafael Pérez (comps.), 
200 años de urbanismo en México. Una mirada a la politica habitacional en México, El Colegio Mexiquense, Zinacantepec, pp. 151-162 (2011); "Adquisición de reservas territoriales para la construcción de vivienda social a través de la Sedesol en el Estado de México", en Alfonso Iracheta y Enrique Soto (comps.), Impacto de la vivienda en el desarrollo urbano. Una mirada a la política habitacional en México, El Colegio Mexiquense, Zinacantepec, México, pp. 351-366 (2009). 